\title{
Penetrating Pancreatic Injury
}

\author{
Lillian DiGiacomo • Martin A. Schreiber
}

Published online: 11 April 2015

(C) Springer International Publishing AG 2015

\begin{abstract}
Pancreatic injury following penetrating trauma is a rare but complex problem in trauma surgery. The retroperitoneal location of the pancreas and its intricate anatomic relationship to other vital structures both contribute to this complexity. Accurate intraoperative diagnosis of pancreatic injury can be difficult, making careful and thorough operative exposure paramount. Operative management is guided by the presence of associated injuries and the severity of the pancreatic injury. External drainage, resection, and staged reconstruction when indicated are the pillars of operative management for the majority of injuries. Post-injury complications are common and can include fistula, pancreatitis, abscess, hemorrhage, and pseudocyst. ERCP is central to the management of these complications and can aid in avoiding return to the operating room. Because of the significant morbidity and mortality associated with penetrating pancreatic injury, collaboration among surgical disciplines is warranted to optimize outcome.
\end{abstract}

Keywords Pancreatic trauma $\cdot$ Pancreatic duct injury . Pancreaticoduodenectomy $\cdot$ Pseudocyst $\cdot$ ERCP

This article is part of the Topical Collection on Penetrating Injuries to Solid Abdominal Viscera

L. DiGiacomo $\cdot$ M. A. Schreiber $(\bowtie)$

Division of Trauma, Critical Care \& Acute Care Surgery, Oregon Health \& Science University, L611 3181 SW Sam Jackson Park

Road, Portland, OR 97239-3098, USA

e-mail: schreibm@ohsu.edu

L. DiGiacomo

e-mail: lillian.digiacomo@kp.org

L. DiGiacomo

Kaiser Permanente South Sacramento Medical Center, 6600

Bruceville Road, Sacramento, CA 95823, USA

\section{Introduction}

Pancreatic injury remains one of the most challenging problems in trauma surgery. Penetrating injury to the pancreas is rare, can be difficult to diagnose, and commonly occurs in the setting of other life-threatening injuries. Persistently high mortality and morbidity in pancreatic injury has prompted ongoing investigations of management protocols and outcomes. This review outlines the common management strategies for different grades of injury and provides a survey of the most pervasive complications and their possible interventions.

Presentation of Penetrating Pancreatic Injury

Of all abdominal trauma, pancreatic injury occurs in approximately $4 \%[1,2 \cdot \bullet]$. Of these, approximately $70 \%$ are the result of penetrating injury $[1,3,4 \cdot]$. The pancreas is injured in $6 \%$ of abdominal gunshot wounds and $2 \%$ of abdominal stab wounds [5]. Penetrating pancreatic injury almost uniformly occurs in the presence of other organ injury, complicating the presentation and management of these injuries $[5,6 \bullet, 7]$. Combination injury is a key factor in the presentation of these rare injuries, as major adjacent organ injury and the associated blood loss often result in early death [3, $5,6 \bullet, 7]$. Close to $50 \%$ of patients will arrive to the emergency department in shock despite appropriate resuscitative efforts from pre-hospital personnel $[6 \bullet, 8 \bullet]$. Additionally, pancreatic trauma is commonly accompanied by postoperative complications, many of which can result in late death $[3,6 \bullet, 7]$. Therefore, these cases overwhelmingly require directed damage control maneuvers and early subspecialist involvement to reduce both early and late mortality. 
Common Injuries Associated with Penetrating Pancreatic Injury

Injury to the pancreas is considered one of the most complex wounds in trauma, owing to its complicated anatomical association with multiple other organs $[5,7,9]$. Its retroperitoneal location can make pancreatic injury difficult to detect [9]. Studies have consistently demonstrated concurrent associated injury in two to four other organs, and greater than $90 \%$ of cases are associated with additional intraabdominal injury to organs such as the liver, spleen, stomach, diaphragm, duodenum, common bile duct, and colon $[1,4 \bullet, 5,9-11]$. As expected, proximal pancreatic injury is often associated with duodenal injury, while distal pancreatic injury is commonly associated with splenic injury [12••].

Pancreatic injury is associated with vascular injury in approximately one third of patients $[1,7,12 \bullet \bullet]$. The aorta, renal vessels, portal vein, IVC, and splenic vessels are at particular risk for injury, significantly increasing both the morbidity and (early) mortality associated with pancreatic injury $[3,6 \bullet, 10$, $12 \bullet \bullet, 13]$.

\section{Diagnosis of Penetrating Pancreatic Injury}

The retroperitoneal location of the pancreas is protective but also complicates diagnosis $[12 \bullet \bullet, 14]$. In penetrating trauma, concern for pancreatic injury may be evident based upon location of the external wounds. In the trauma resuscitation bay, plain abdominal radiographs may suggest a missile trajectory concerning for pancreatic involvement [15]. Plain films can rarely demonstrate retroperitoneal air bubbles that may suggest an injury to the pancreaticoduodenal complex [13]. Laboratory tests such as serum amylase and lipase have been shown to be of little use in accurately detecting injury to the pancreas $[1,5,7,14]$. Some studies have confirmed normal serum amylase levels in patients with injuries as severe as complete pancreatic transection, highlighting the lack of correlation between injury severity and serum amylase [13].

Penetrating injury to the abdomen is generally managed with immediate exploration in the operating room without pause for extensive imaging. For the few penetrating trauma patients who might undergo imaging prior to proceeding to the operating room, computed tomography (CT) with intravenous iodinated contrast is the imaging modality of choice [5]. CT can demonstrate fracture or laceration of the pancreas, intraparenchymal hematoma, or peripancreatic fluid or edema. Secondary signs of pancreatic trauma include peripancreatic fat stranding, fluid between the pancreas and the splenic vein, and thickening of the left anterior pararenal fascia [5, 7, 15].

CT has demonstrated a sensitivity and specificity of more than $85 \%$ for pancreatic injury [16]. In the remainder of cases, radiographic changes to the pancreas are delayed and may not be visible on $\mathrm{CT}$ obtained promptly post-injury $[7,15]$. In particular, an early pancreatic transection can be hard to identify while the freshly lacerated edges remain closely apposed. Accumulation of low-attenuation fluid or hematoma between the two edges allows diagnosis only after a delay. Therefore, a select number of cases may benefit from repeat imaging 12 to 24 hours following injury [7].

In contrast, $\mathrm{CT}$ is a poor modality for the determination of pancreatic duct disruption. Several studies have suggested accuracy as low as $40 \%$ for CT identification of duct injury [15]. In a study from our institution, recent improvements in CT technology were shown to increase the sensitivity and specificity of CT for duct injury [17]. Duct disruption is the major factor in management of pancreatic injury, and intraoperative determination of this with either endoscopic or operative pancreatography is often necessary. This is discussed below, "Injury to the Pancreatic Duct." Because accurate determination of the involvement of the duct is important in grading severity of pancreatic injury, injury scores based on CT are often underestimated (Table 1) [7].

Operative Exploration and Management of Pancreatic Injury

Clinical management of penetrating injury to the abdomen includes initial resuscitation according to Advanced Trauma Life Support guidelines. The great majority of patients with penetrating injury to the abdomen proceed directly to the operating room; thus, penetrating pancreatic injury is primarily an intraoperative diagnosis. Once in the operating room, the principles of operative management include immediate control of intraabdominal bleeding, closure of hollow viscus perforation to control peritoneal contamination, and prompt volume replacement to prevent worsening coagulopathy, acidosis, and hypothermia $[5,6 \bullet, 7]$. This damage control technique is then followed by either immediate and/or delayed treatment as dictated by the severity of the pancreatic injury, as well as the physiologic status of the patient $[10,16]$. The skill set of the surgeon is also important to surgical decision-making in these patients.

In the patient who has sustained penetrating injury to the abdomen, a number of key indicators will signal pancreatic

Table 1 AAST grading of pancreatic injury [18]

\begin{tabular}{ll}
\hline Grade & Description \\
\hline I & Minor contusion or laceration without duct injury \\
II & Major contusion or laceration without duct injury \\
III & $\begin{array}{l}\text { Transection or major laceration with duct disruption } \\
\text { in distal pancreas }\end{array}$ \\
IV & $\begin{array}{c}\text { Transection of proximal pancreas or major laceration } \\
\text { with associated injury to the ampulla }\end{array}$ \\
V & Massive disruption of the pancreatic head \\
\hline
\end{tabular}


injury to the trauma surgeon. Close inspection should be made for retroperitoneal hematoma, bile staining, fat saponification, and lesser sac fluid or edema [19•]. Complete inspection of the pancreas should be made when any of these indicators are discovered $[5,7,16]$. Thorough investigation of the pancreas involves entry into the lesser sac via the gastrocolic omentum, medial rotation of the hepatic flexure of the colon, kocherization of the duodenum, and mobilization of the spleen and splenic flexure. These maneuvers will allow good visualization of the anterior surface of the pancreas.

Visualization of the posterior surface requires dissection into the retropancreatic space with careful elevation of the pancreas off of the splenic vessels [19•]. This is usually achieved either through division of the retroperitoneal attachments along the inferior border of the pancreas or by mobilizing the spleen, tail, and body of the pancreas off of the underlying kidney $[5,7,13]$. Such a mobilization can permit bimanual palpation of the pancreas. In some instances, the physiologic status of the critically injured patient may prohibit such thorough exploration [16]. In penetrating trauma, an attempt should be made to explore the entire missile tract $[13,19 \bullet]$.

Once adequate exposure of the pancreas has been gained, management of pancreatic injury depends largely on grade and anatomic location (Table 1) [11]. Determination of the presence of ductal injury is of paramount importance and is addressed in a separate section below, "Injury to the Pancreatic Duct." Drainage is the key element of surgical management, regardless of grade or location $[10,11,13]$. The majority of injuries are Grade I and II injuries, which can be managed with local debridement of devitalized tissue and closed suction drainage $[1,6 \bullet, 10$, 12••, 16]. Primary repair of any capsular tear has been shown to result in either necrosis or subsequent pseudocyst and should be avoided [1, 13]. Using a piece of viable omentum to buttress a parenchymal injury that does not involve the duct is an effective way of preventing further pancreatic necrosis [5]. Closed suction drainage should likewise accompany omental plugging, for control of any fistula that may develop from a minor duct injury.

Grade III injuries generally require distal pancreatectomy (Fig. 1). In the stable patient, splenic salvage should be considered [5]. In the critical adult trauma patient, concomitant splenectomy is advisable in order to minimize bleeding that can result from dissection of the distal pancreas [13, 16, 19•]. Isolating and dividing the splenic vessels 1 to $2 \mathrm{~cm}$ either proximal or distal to the ductal injury can protect ligated vessels from the fluid leakage of a potential postoperative fistula $[5,19 \bullet]$. Distal pancreatectomy and splenectomy are again accompanied by closed-suction drainage. In contrast to adults, efforts are generally made to preserve the spleen in trauma to the pediatric pancreas, given the increased incidence of postsplenectomy complications in this population $[1,5,13,20]$.

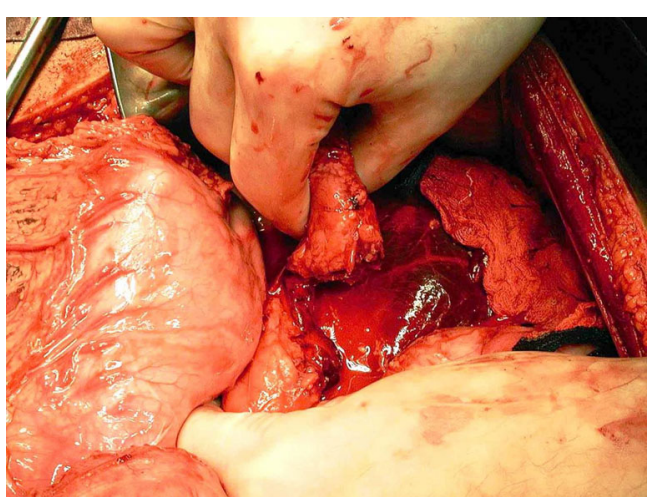

Fig. 1 Grade III pancreatic injury. (Photo credit: MA Schreiber)

Rarely, the distal pancreas may be preserved with Roux-en$Y$ reconstruction of the distal portion and closure of the proximal stump [1, 9, 16]. This option is particularly desirable in patients whose injury would necessitate large volume loss of the pancreas, resulting in endocrine deficiency [20]. When performed in the critically ill patient, Roux-en-Y distal pancreaticojejunostomy poses the often intolerable risk of a new anastomosis. Therefore, salvage of the distal pancreas should only be attempted in the stable patient without other serious injuries [5].

The need to close the pancreatic stump following distal pancreatectomy represents a long-standing debate which has been explored in both the trauma and hepatobiliary literature [21]. Current data support suture ligation of the pancreaticoduodenal arteries and exposed duct, with oversewing or stapling of the cut edge of the pancreatic parenchyma $[7,12 \bullet \bullet, 13,19 \bullet]$. An excessively tight suture closure can result in pancreatic necrosis, which later precipitates fistula or pseudocyst. Staples are often used in an attempt to avoid suturing. Alternatively, a "fish mouth" closure has also been proposed in which the anterior and posterior cut surfaces of the pancreatic stump are fashioned in an oblique plane such that they may be apposed to each other with running or mattress sutures [5]. As discussed above, another technique which has proven effective for prevention of stump leakage is omental plugging of the pancreatic stump [8•]. This is thought to act as a seal for small pancreatic ducts not sealed by staples. Notwithstanding these many options, no particular method has been definitively proven superior to another $[5,11]$.

Single-stage external drainage with or without distal resection of the pancreas will be adequate management for the majority of pancreatic injuries. In contrast to lower grade injuries, Grade IV and V injuries are more complex, almost always require a staged procedure, and their management may benefit from the input of a hepatobiliary specialist. Grade IV injuries can respond well to external drainage, with a large proportion demonstrating spontaneous resolution of the ensuing fistula [13]. For grade V injuries, the safest approach has been shown to be initial washout and drainage, followed by 
delayed pancreaticoduodenectomy to treat pancreatic head disruption $[7,13,15,16]$. Suggested indications for this approach include severe damage to the head of the pancreas or duodenum such that primary repair is not possible; disruption of the duct at the level of the pancreatic head with associated injury to the duodenum and common bile duct; disruption of the ampulla of Vater; bleeding from the head of the pancreas which cannot be controlled; or retropancreatic injury to the portal or mesenteric vessels $[5,7]$. Pancreaticoduodenectomy is estimated to be required in 3 to $5 \%$ of traumatic pancreatic injuries $[4 \cdot, 12 \bullet \bullet]$. Performing a Whipple procedure in a trauma patient has been canonized as a procedure to be undertaken "only when the dissection has already been done for you."

Large-volume retropancreatic bleeding often requires a prompt Kocher maneuver with immediate transection of the neck of the pancreas for access to the portomesenteric system posterior to it [7]. This is followed by manual compression for immediate control and then attempted repair. Some may require immediate pancreatic resection in order to control ongoing hemorrhage [10]. Similar to other operations for multiplyinjured trauma patients, damage control surgery with delay in definitive repair of high-grade pancreatic injuries is indicated for patients who present with coagulopathy, hypothermia, acidosis, ongoing hemodynamic instability, or massive blood product transfusion requirement. The morbidity and mortality associated with complex reconstruction are prohibitive, and simple closed suction drainage may be all that is possible in the initial operation [5].

Alternatively, if the patient can tolerate a resection during their first operation, transection of the stomach, jejunum, and pancreatic stump can be performed during damage control laparotomy, with reconstruction completed at a subsequent laparotomy, usually 48 hours later $[5,6 \bullet, 10,12 \bullet \cdot$. Delaying reconstruction is thought to result in a firmer pancreas, a dilated common bile duct, and less edematous bowel, all of which may facilitate a more reliable reconstruction $[4 \bullet, 19 \bullet]$. In contrast to the elective Whipple procedure, both the uncinate process and the gallbladder may be preserved in the Whipple procedure performed for trauma, simplifying the operation $[13,19 \bullet]$.

These severe injuries are rare. Because they almost exclusively occur in patients with multiple other injuries, mortality rates associated with this operation have been reported to be approximately $35 \%$, and postoperative complications occur in the majority of those who do survive $[5,7,12 \bullet \cdot$. As expected, shock on presentation and multiple associated injuries are both predictors of mortality in patients with high-grade injuries. In high-grade pancreatic injury, placement of a either a silastic nasojejunal tube or feeding jejunostomy is an additional maneuver which should be considered intraoperatively, due to possible difficulty with enteral feeding in the postoperative period (see "Post-operative Management of Pancreatic Injury" below).
Injury to the Pancreatic Duct

The presence or absence of injury to the pancreatic duct is considered the key characteristic of pancreatic injury and is critical to both prevention of complications and prognosis [1, $5,13,15]$. Duct disruption occurs in approximately $15 \%$ of pancreatic injuries. Failed, incomplete, or delayed recognition of pancreatic duct injury in the setting of pancreatic trauma are major contributors to subsequent morbidity $[5,7,13,15]$. Leakage of pancreatic enzymes results in peripancreatic inflammation that can precipitate post-injury sepsis, the main factor in late mortality in these patients.

In penetrating trauma, most surgeons suggest direct examination with loupe magnification to identify pooling of clear pancreatic fluid in the area of a suspected injury $[5,13]$. Previously identified intraoperative criteria can also facilitate the recognition of ductal injury. These are: direct visualization of ductal disruption, complete transection of the pancreas, laceration of more than half the diameter of the pancreas, central perforation, and severe maceration of the gland $[19 \bullet, 22 \cdot \bullet]$. Intraoperative ultrasound may be helpful in identifying the extent of a parenchymal laceration, though this methodology has not been widely examined in trauma [13]. Similarly, injury to the duodenum must be excluded with a thorough search $[22 \bullet \bullet]$.

Cholangiopancreatography is the most widely accepted modality for definitive determination of ductal disruption [15]. For penetrating injury, cholangiopancreatography can be done intraoperatively, either with an open technique or endoscopically. Extravasation of contrast under fluoroscopy following varied cannulation approaches can demonstrate duct disruption. Cannulation of the cystic duct following cholecystectomy yields good visualization of the distal common bile duct, ampulla, and pancreatic duct [13]. Alternatively, the gallbladder or common bile duct may be accessed with a 25 gauge needle, or the pancreatic duct may be cannulated directly via the ampulla following duodenotomy $[7,13]$. This latter technique is discouraged because it converts an isolated pancreatic injury into a combined pancreaticoduodenal injury. All of these techniques can provide fluoroscopic visualization of the distal common bile duct, ampulla, and pancreatic duct (via retrograde reflux of contrast). In patients with distal transection, the distal duct can also be cannulated directly to confirm the condition of the proximal duct and the ampulla. Cannulation of the duct at this level can be particularly challenging due its diminutive size $[5,13]$.

In the absence of fluoroscopy, gentle cannulation of the pancreatic duct with a blunt probe via the ampulla may also indicate the proximity of the duct to the external injury. Similarly, gentle saline irrigation via a $5 \mathrm{Fr}$ catheter placed through the ampulla of Vater may allow injury to be recognized in the absence of fluoroscopy [22••]. Failing definitive identification of ductal injury, many surgeons recommend empiric distal 
pancreatectomy if suspicion of injury is high, versus external drainage accompanied by postoperative endoscopic retrograde cholangiopancreatography (ERCP) if suspicion of injury is low $[11,13]$.

Intraoperative ERCP has been reported but is often difficult to utilize safely and accurately in the acutely injured trauma patient [7, 13]. Magnetic resonance cholangiopancreatography (MRCP) is also a reliable and accurate imaging modality for identification of pancreatic duct and surrounding injuries [7]. It can be done rapidly and without contrast material, but it is rarely a feasible imaging modality for the penetrating trauma patient who can seldom be diverted from their operative trajectory.

\section{Postoperative Management of Pancreatic Injury}

When damage control maneuvers are performed by a trauma surgeon in the urgent setting, the need for referral to a hepatobiliary surgeon at a tertiary center should always be considered for further management [2••]. In the immediate postoperative period, attention should be paid to total body fluid balance as well as drain output and character, as patients can require ongoing volume resuscitation due to these losses [7]. Ileus and feeding difficulty are other common challenges. Elemental tube feeding formulas are thought to provoke less pancreatic stimulation and may be better tolerated [7]. Postoperative pancreatic insufficiency can result in both endocrine and exocrine disorders and may require long-term treatment.

\section{Complications of Pancreatic Injury}

Pancreas-related complications occur in approximately one third of patients experiencing penetrating pancreatic injury $[1,3,7,11]$. Complications following this type of injury are largely due to either a delay in diagnosis or incomplete treatment of the injury $[8 \bullet, 10,15]$. The grade of the injury has been found to have a significant effect on the risk of ensuing complication and mortality, while infectious complications appear to occur at the same rate regardless of the type of operative management performed $[5,6 \bullet, 11]$. Later development of a pancreatic complication is the major contributor to the morbidity and mortality associated with pancreatic injury [3]. Fortunately, the majority of complications can be managed without repeat operation $[5,9,12 \bullet \bullet$. Across various types of complications, ERCP is essential to diagnosis and optimal management, and can decrease the need for repeat laparotomy $[13,23 \bullet \bullet]$.

The most common complication is pancreatic fistula, occurring in approximately 15 to $30 \%$ of injuries [1, 3, 6• 15]. Fistula is commonly resolved with external drainage alone. ERCP and stenting of the pancreatic duct can be effective for recalcitrant leaks, such as those with greater than $500 \mathrm{ml}$ per day output persisting for greater than 1 week [7, 16, 23••].
ERCP with sphincterotomy alone (without stenting) has also been described $[6 \bullet, 8 \bullet$.

Octreotide may be a useful adjunct for decreasing output in post-traumatic pancreatic fistula, though data has not definitely confirmed its effectiveness in fistula closure [1, 5, 7]. In the majority of patients, spontaneous fistula closure occurs within 6 to 8 weeks following external drainage $[5,6 \bullet, 11]$. For those that do not resolve in this timeframe, repeat laparotomy, resection (for distal injuries), and/or reconstruction (for proximal injuries) may be required $[5,6 \bullet, 7,16]$. Other important treatment adjuncts include control of concomitant infection and good nutrition [16]. High output fistulas (greater than $500 \mathrm{ml} /$ day) often dictate parenteral nutrition, while low output fistulas can often be managed with enteral nutrition [5, 16]. In either case, maintaining adequate alimentation in the injured patient is essential to fistula closure [13].

Postoperative pancreatitis occurs in up to $18 \%$ [13]. This may develop as a result of an unrecognized pancreatic duct injury and can be identified by elevated serum lipase [16]. Posttraumatic pancreatitis is a result of fibrosis and stricture of the duct [5]. This generally only requires supportive care, with resolution occurring within a few weeks. If pancreatic necrosis ensues, re-exploration and pancreatic debridement are required. Large bore irrigation and external drainage catheters may be placed for ongoing removal of non-viable tissue [7].

Pancreatic abscess formation occurs in 10 to $25 \%$ and is frequently associated with hollow viscus, solid organ, or pancreatic duct injury $[3,5,13]$. Abscess should be suspected in instances of prolonged postoperative ileus, fever, leukocytosis, or unexplained abdominal tenderness [7]. This complication is treated with CT-guided external drainage. Fluid should be sent to the laboratory for determination of amylase level and microbial content. Broad spectrum antibiotics should be initiated until speciation returns. When percutaneous aspiration is unsuccessful, pancreatic abscess can rarely require open surgical drainage $[5,6 \bullet, 16]$.

Secondary hemorrhage results from autodigestion of surrounding tissues by leaking pancreatic fluid and is a complication that can rapidly progress to death $[6 \bullet, 7,12 \bullet \bullet]$. First-line treatment is radiographic embolization, but relaparotomy is often necessary $[1,13]$. Ongoing leakage of pancreatic enzymes may similarly contribute to anastomotic breakdown. One study found as many as $25 \%$ of new bowel anastomoses failed in the setting of concomitant management of pancreatic injury [12••].

Pancreatic pseudocyst presents late in the course of disease (weeks to months after injury), and the natural history depends mainly on the involvement of the pancreatic duct [11]. For the purposes of management, trauma patients who develop a pancreatic pseudocyst should be assumed to have a missed ductal injury until proven otherwise $[5,7,15]$. This is best determined by MRCP or ERCP $[9,13]$. If the duct is not involved, 
percutaneous drainage may be sufficient [13]. Disruption of the duct almost uniformly requires endoscopic stenting $[1,7,16]$. In those resulting from a distal duct injury, persistent or recurrent pseudocysts which do not resolve with external drainage may be treated with distal pancreatectomy [5]. Cystogastrostomy, cystoduodenostomy, or cystojejunostomy are options for large symptomatic pancreatic pseuodocysts with mature walls. These can be performed either endoscopically or surgically [9, $16,23 \cdot \bullet$. Such internal drainage procedures have been found to have a lower incidence of subsequent infectious complications or pseudocyst recurrence [9]. However, there is little long-term data in trauma patients on the successful use of endoscopic cyst-enteric drainage. A small proportion of pseudocysts may resolve spontaneously $[5,9]$.

\section{Outcomes Following Pancreatic Injury}

In addition to the pancreas-specific complications described above, these patients are at risk for a multitude of posttraumatic outcomes that result in delayed death. Sepsis, multiple organ failure, acute respiratory distress syndrome (ARDS), and small bowel ischemic insult as a result of concomitant mesenteric vascular injury or thrombosis due to pancreatitis are common $[7,8 \bullet]$. The common necessity for repeat laparotomy due to intraabdominal sepsis also contributes to operative risk and all-cause mortality [8•]. In a retrospective review of 219 civilian gunshot injuries to the pancreas, shock on presentation, presence of vascular injury, grade of pancreatic injury, and presence of proximal (versus distal) pancreatic injury were all significant predictors of mortality [6•]. Unrecognized pancreatic duct disruption is a major cause of morbidity and mortality in this group of patients $[5,7]$. Overall pancreas-specific mortality is reported to be approximately 20 to $30 \%$, and more than half of these deaths occur in patients with significant vascular injury $[6 \bullet 11,12 \bullet \bullet, 22 \bullet \bullet]$.

\section{Conclusion}

Pancreatic injury is rare in penetrating trauma. Its unique anatomy can both obscure the diagnosis of injury and make its treatment treacherous. Methodical management with damage control techniques, wide external drainage, thorough interrogation of ductal integrity, and delayed reconstruction are essential to an optimal outcome. In addition, vigilant postoperative observation and an attempt at conservative support of post-injury complications have been shown to be effective. Penetrating pancreatic injury is among the most challenging problems in trauma surgery and requires an informed and comprehensive approach with multidisciplinary participation.

\section{Compliance with Ethics Guidelines}

Conflict of Interest Lillian DiGiacomo and Martin A. Schreiber declare that they have no conflicts of interest.

Human and Animal Rights and Informed Consent This article does not contain any studies with human or animal subjects performed by any of the authors.

\section{References}

Papers of particular interest, published recently, have been highlighted as:

- Of importance

•. Of major importance

1. Lahiri R, Bhattacharya S. Pancreatic trauma. Ann R Coll Surg Engl. 2013;95:241-5.

2.• Ragulin-Coyne E, Witkowski ER, Chau Z, et al. National trends in pancreaticoduodenal trauma: interventions and outcomes. HPB. 2014;16:275-81. This article utilizes data from the US Nationwide Inpatient Sample to investigate trends in pancreaticoduodenal injury management over the last decade and to correlate those trends with recent outcomes for injured patients. Their results demonstrated that while non-operative management of pancreaticoduodenal trauma has increased, mortality from pancreaticoduodenal injury has simultaneously decreased. Post-operative morbidity has also increased, and penetrating trauma was identified as a significant predictor of complications.

3. Recinos G, DuBose JJ, Teixeira PGR, et al. Local complications following pancreatic trauma. Injury. 2009;40:516-20.

4. Thompson CM, Shalhub S, DeBoard ZM, et al. Revisiting the pancreaticoduodenectomy for trauma: a single institution's experience. J Trauma Acute Care Surg. 2013;75:225-8. This paper describes one of the largest series of patients who underwent Whipple procedure for trauma to the pancreaticoduodenal complex. They demonstrate the lowest mortality rate to date in this subpopulation (13\% for the initial hospitalization). They attribute this success to their use of initial damage control surgery followed by staged reconstruction, as opposed to single-stage pancreaticoduodenectomy with immediate reconstruction.

5. Subramanian A, Dente CJ, Feliciano D. The management of pancreatic trauma in the modern era. Surg Clin N Am. 2007;87:1515-32.

6. Chinnery GE, Krige JEJ, Kotze UK, et al. Surgical management and outcome of civilian gunshot injuries to the pancreas. Br J Surg. 2012;99 Suppl 1:140-8. This paper reviews a large series of patients with penetrating pancreatic injuries in order to characterize management strategies and risk factors predictive of outcome. Data collected over 33 years at a level I trauma center demonstrated the high rate of associated organ and vascular injuries, and highlights the role of damage control surgery in complex multiple injury.

7. Krige JEJ, Beningfield SJ, Nicol AJ, et al. The management of complex pancreatic injuries. SAJS. 2005;43:92-102.

8. Krige JEJ, Kotze UK, Nicol AJ, et al. Morbidity and mortality after distal pancreatectomy for trauma: a critical appraisal of 107 consecutive patients undergoing resection at a Level 1 trauma centre. 
Injury. 2014;45:1401-8. This paper provides a rigorous analysis of the post-operative complications associated with the most commonly used surgical intervention for Grade III pancreatic injury, distal pancreatectomy. Mortality was found to be closely correlated with the presence of associated injury, and "surgical simplicity" was highlighted as the key to successful management of these complicated injuries.

9. Buccimazza I, Thomson SR, Anderson F, et al. Isolated main pancreatic duct injuries spectrum and management. Am J Surg. 2006;191:448-52.

10. Seamon MJ, Kim PK, Stawicki SP, et al. Pancreatic injury in damage control laparotomies: is pancreatic resection safe during the initial laparotomy? Injury. 2009;40:61-5.

11. Vasquez JC, Coimbra R, Hoyt DB, et al. Management of penetrating pancreatic trauma: an 11-year experience of a level-1 trauma center. Injury. 2001;32:753-9.

12.• Vertrees A, Elster E, Jindal R, et al. Surgical management of modern combat-related pancreatic injuries: traditional management and unique strategies. Mil Med. 2014;179:315-9. This paper not only brings crucial military data to bear on the clinical decisionmaking involved in managing penetrating pancreatic trauma, but also describes the first successful use of islet cell preservation and auto-transplantation following pancreatic resection for trauma. The protocol for islet cell processing and posttransplantation monitoring is provided in detail.

13. Degiannis E, Glapa M, Loukogeorgakis SP, et al. Management of pancreatic trauma. Injury. 2008;39:21-9.

14. Blatnik J, Ponsky T. Alternative approaches to pancreatic duct injury. SMJ. 2009;102:1199-200.

15. Debi U, Kaur R, Prasad KK, et al. Pancreatic trauma: a concise review. World J Gastroenterol. 2013;19:9003-11.

16. Ahmed N, Vernick JJ. Pancreatic injury. SMJ. 2009;102:1253-6.

17. Tey SH, Sheppard BC, Mullins RJ, et al. Diagnosis and management of blunt pancreatic ductal injury in the era of high-resolution computed axial tomography. Am J Surg. 2007;193:641-3.
18. Moore EE, Cogbill TH, Malangoni M, et al. Scaling system for organ specific injuries. www.aast.org/library/traumatools/ injuryscoringscales.asp.

19. Yilmaz TH, Hauer TJ, Smith MD, et al. Operative techniques in pancreatic trauma - a heuristic approach. Injury. 2013;44:153-5. This article provides a utilitarian summary of the most important intraoperative maneuvers for addressing pancreatic trauma, enabling surgeons unfamiliar with the pancreas to perform effective damage control interventions while preventing some of the most common mishaps and complications.

20. Meier DE, Coln CD, Hicks BA, et al. Early operation in children with pancreas transection. J Pediatr Surg. 2001;36:341-4.

21. Meniconi RL, Caronna R, Borreca D, et al. Pancreato-jejunostomy versus hand-sewn closure of the pancreatic stump to prevent pancreatic fistula after distal pancreatectomy: a retrospective analysis. BMC Surg. 2013;13:23-8.

22.• Asensio JA, Petrone P, Roldan G, et al. Pancreaticoduodenectomy: a rare procedure for the management of complex pancreaticoduodenal injuries. J Am Coll Surg. 2003;197:937-42. This case series reviews the largest group of penetrating pancreatic injuries requiring pancreaticoduodenectomy collected to date. It aims to validate indications for this complex procedure in the critical trauma patient, and notes a high rate of concurrent vascular injury in these patients, often requiring resuscitative thoracotomy. They report a mortality rate of approximately $35 \%$ despite a high mean Injury Severity Score.

23.• Thomson DA, Krige JEJ, Thomson SR, et al. The role of endoscopic retrograde pancreatography in pancreatic trauma: a critical appraisal of 48 patients treated at a tertiary institution. J Trauma Acute Care Surg. 2014;76:1362-6. This retrospective review of consecutive pancreatic injuries over 28 years highlights the use of ERCP to diagnose and treat post-injury complications. Importantly, this data supports decreased operative intervention for many types of complications when aggressive endoscopic treatments are used selectively and effectively. 